\title{
Research Article \\ General Helices of AW(k)-Type in the Lie Group
}

\author{
Dae Won Yoon \\ Department of Mathematics Education and RINS, Gyeongsang National University, \\ Jinju 660-701, Republic of Korea \\ Correspondence should be addressed to Dae Won Yoon, dwyoon@gnu.ac.kr
}

Received 13 October 2012; Accepted 2 December 2012

Academic Editor: Hui-Shen Shen

Copyright (C) 2012 Dae Won Yoon. This is an open access article distributed under the Creative Commons Attribution License, which permits unrestricted use, distribution, and reproduction in any medium, provided the original work is properly cited.

We study curves of AW(k)-type in the Lie group $G$ with a bi-invariant metric. Also, we characterize general helices in terms of $A W(k)$-type curve in the Lie group $G$.

\section{Introduction}

The geometry of curves and surfaces in a 3-dimensional Euclidean space $\mathbb{R}^{3}$ represented for many years a popular topic in the field of classical differential geometry. One of the important problems of the curve theory is that of Bertrand-Lancret-de Saint Venant saying that a curve in $\mathbb{R}^{3}$ is of constant slop; namely, its tangent makes a constant angle with a fixed direction if and only if the ratio of torsion $\tau$ and curvature $\kappa$ is a constant. These curves are said to be general helices. If both $\tau$ and $\kappa$ are nonzero constants, the curve is called cylindrical helix. Helix is one of the most fascinating curves in science and nature. Scientists have long held a fascinating, sometimes bordering on mystical obsession for helical structures in nature. Helices arise in nanosprings, carbon nanotubes, $\alpha$-helices, DNA double and collagen triple helix, the double helix shape is commonly associated with DNA, since the double helix is structure of DNA.

The problem of Bertrand-Lancret-de Saint Venant was generalized for curves in other 3-dimensional manifolds-in particular space forms or Sasakian manifolds. Such a curve has the property that its tangent makes a constant angle with a parallel vector field on the manifold or with a Killing vector field, respectively. For example, a curve $\alpha(s)$ in a 3-dimensional space form is called a general helix if there exists a Killing vector field $V(s)$ with constant length along $\alpha$ and such that the angle between $V$ and $\alpha^{\prime}$ is a non-zero constant (see [1]). A general helix defined by a parallel vector field was studied in [2]. Moreover, in [3] it is shown that general helices in a 3-dimensional space form are extremal curvatures of a functional involving a linear combination of the curvature, the torsion, and a constant. General helices also called the Lancret curves are used in many applications (e.g., [4-7]). 
The notion of AW(k)-type submanifolds was introduced by Arslan and West in [8]. In particular, many works related to curves of $A W(k)$-type have been done by several authors. For example, in $[9,10]$ the authors gave curvature conditions and charaterizations related to these curves in $\mathbb{R}^{n}$. Also, in [11] they investigated curves of $A W(k)$ type in a 3-dimensional null cone and gave curvature conditions of these kinds of curves. However, to the author's knowledge, there is no article dedicated to studying the notion of AW(k)-type curves immersed in Lie group.

In this paper, we investigate curvature conditions of curves of $A W(k)$-type in the Lie group $G$ with a bi-invariant metric. Moreover, we characterize general helices of AW(k)-type in the Lie group $G$.

\section{Preliminaries}

Let $G$ be a Lie group with a bi-invariant metric $\langle$,$\rangle and D$ the Levi-Civita connection of the Lie group $G$. If $\mathfrak{g}$ denotes the Lie algebra of $G$, then we know that $\mathfrak{g}$ is isomorphic to $T_{e} G$, where $e$ is identity of $G$. If $\langle$,$\rangle is a bi-invariant metric on G$, then we have

$$
\begin{gathered}
\langle X,[Y, Z]\rangle=\langle[X, Y], Z\rangle, \\
D_{X} Y=\frac{1}{2}[X, Y]
\end{gathered}
$$

for all $X, Y, Z \in \mathfrak{g}$.

Let $\alpha: I \subset \mathbb{R} \rightarrow G$ be a unit speed curve with parameter $s$ and $\left\{V_{1}, V_{2}, \ldots, V_{n}\right\}$ an orthonrmal basis of $\mathfrak{g}$. In this case, we write that any vector fields $W$ and $Z$ along the curve $\alpha$ as $W=\sum_{i=1}^{n} w_{i} V_{i}$ and $Z=\sum_{i=1}^{n} z_{i} V_{i}$, where $w_{i}: I \rightarrow \mathbb{R}$ and $z_{i}: I \rightarrow \mathbb{R}$ are smooth functions. Furthermore, the Lie bracket of two vector fields $W$ and $Z$ is given by

$$
[W, Z]=\sum_{i=1}^{n} w_{i} z_{j}\left[V_{i}, V_{j}\right]
$$

Let $D_{\alpha^{\prime}} W$ be the covariant derivative of $W$ along the curve $\alpha, V_{1}=\alpha^{\prime}$, and $W^{\prime}=\sum_{i=1}^{n} w_{i}^{\prime} V_{i}$, where $w_{i}^{\prime}=d w_{i} / d s$. Then we have

$$
D_{\alpha^{\prime}} W=W^{\prime}+\frac{1}{2}\left[V_{1}, W\right]
$$

A curve $\alpha$ is called a Frenet curve of osculating order $d$ if its derivatives $\alpha^{\prime}(s), \alpha^{\prime \prime}(s)$, $\alpha^{\prime \prime \prime}(s), \ldots, \alpha^{(d)}(s)$ are linearly dependent and $\alpha^{\prime}(s), \alpha^{\prime \prime}(s), \alpha^{\prime \prime \prime}(s), \ldots, \alpha^{(d+1)}(s)$ are no longer linearly independent for all $s$. To each Frenet curve of order $d$ one can associate an orthonormal $d$-frame $V_{1}(s), V_{2}(s), V_{3}(s), \ldots, V_{d}(s)$ along $\alpha$ (such that $\alpha^{\prime}(s)=V_{1}(s)$ ) called the Frenet frame 
and the functions $k_{1}, k_{2}, \ldots, k_{d-1}: I \rightarrow \mathbb{R}$ said to be the Frenet curvatures, such that the Frenet formulas are defined in the usual way:

$$
\begin{aligned}
D_{V_{1}} V_{1}(s)= & k_{1}(s) V_{2}(s), \\
D_{V_{1}} V_{2}(s)= & -k_{1}(s) V_{1}(s)+k_{2}(s) V_{3}(s), \\
& \vdots \\
D_{V_{1}} V_{i}(s)= & -k_{i-1}(s) V_{i-1}(s)+k_{i}(s) V_{i+1}(s), \\
D_{V_{1}} V_{i+1}(s)= & -k_{i}(s) V_{i}(s) .
\end{aligned}
$$

If $\alpha: I \rightarrow G$ is a Frenet curve of osculating order 3 in $G$, then we define

$$
\bar{k}_{2}(s)=\frac{1}{2}\left\langle\left[V_{1}, V_{2}\right], V_{3}\right\rangle .
$$

Proposition 2.1. Let a be a Frenet curve of osculating order 3 in $G$. Then one has

$$
\begin{aligned}
& {\left[V_{1}, V_{2}\right]=\left\langle\left[V_{1}, V_{2}\right], V_{3}\right\rangle V_{3}=2 \bar{k}_{2} V_{3},} \\
& {\left[V_{1}, V_{3}\right]=\left\langle\left[V_{1}, V_{3}\right], V_{2}\right\rangle V_{2}=-2 \bar{k}_{2} V_{2},} \\
& {\left[V_{2}, V_{3}\right]=\left\langle\left[V_{2}, V_{3}\right], V_{1}\right\rangle V_{1}=2 \bar{k}_{2} V_{1} .}
\end{aligned}
$$

Proof. Let $\alpha$ be a Frenet curve of osculating order 3 with the Frenet frame $\left\{V_{1}, V_{2}, V_{3}\right\}$. Since $\left[V_{1}, V_{2}\right]=a_{1} V_{1}+a_{2} V_{2}+a_{3} V_{3}$, taking the inner product with $V_{1}, V_{2}$, and $V_{3}$, respectively, we have $a_{1}=a_{2}=0$ and $\left\langle\left[V_{1}, V_{2}\right], V_{3}\right\rangle=a_{3}$. Thus, we find

$$
\left[V_{1}, V_{2}\right]=\left\langle\left[V_{1}, V_{2}\right], V_{3}\right\rangle V_{3}
$$

From (2.5), we get

$$
\left[V_{1}, V_{2}\right]=2 \bar{k}_{2} V_{3}
$$

By using the above similar method, we can obtain $\left[V_{1}, V_{3}\right]=-2 \bar{k}_{2} V_{2}$ and $\left[V_{2}, V_{3}\right]=2 \bar{k}_{2} V_{1}$.

Remark 2.2. Let $G$ be a 3-dimensional Lie group with a bi-invariant metric. Then it is one of the Lie groups $S O(3), S^{3}$ or a commutative group, and the following statements hold (see $[6,12])$.

(i) If $G$ is $S O(3)$, then $\bar{k}_{2}(s)=1 / 2$.

(ii) If $G$ is $S^{3} \cong S U(2)$, then $\bar{k}_{2}(s)=1$.

(iii) If $G$ is a commutative group, then $\bar{k}_{2}(s)=0$. 
Proposition 2.3. Let a be a Frenet curve of osculating order 3 in G. Then one has

$$
\begin{aligned}
\alpha^{\prime}(s) & =V_{1}(s), \\
\alpha^{\prime \prime}(s) & =k_{1}(s) V_{2}(s), \\
\alpha^{\prime \prime \prime}(s) & =-k_{1}^{2}(s) V_{1}(s)+k_{1}^{\prime}(s) V_{2}(s)+k_{1}(s) \tau_{1}(s) V_{3}(s), \\
\alpha^{\prime \prime \prime \prime}(s) & =-3 k_{1}(s) k_{1}^{\prime}(s) V_{1}(s)+\left[k_{1}^{\prime \prime}(s)-k_{1}^{3}(s)-k_{1}(s) k_{2}^{2}(s)+2 k_{1}(s) k_{2}(s) \bar{k}_{2}(s)\right. \\
& \left.-k_{1}(s) \bar{k}_{2}^{2}(s)\right] V_{2}(s)+\left(2 k_{1}^{\prime}(s) \tau_{1}(s)+k_{1}(s) \tau_{1}^{\prime}(s)\right) V_{3}(s),
\end{aligned}
$$

where $\tau_{1}(s)=k_{2}(s)-\bar{k}_{2}(s)$.

Proof. Let $\alpha$ be a Frenet curve of osculating order 3 in G. Then we have

$$
\alpha^{\prime \prime}(s)=\frac{d^{2} \alpha}{d s^{2}}=V_{1}^{\prime}(s)=D_{V_{1}} V_{1}(s)-\frac{1}{2}\left[V_{1}(s), V_{1}(s)\right]=k_{1}(s) V_{2}(s)
$$

This implies that

$$
\begin{aligned}
\alpha^{\prime \prime \prime}(s) & =k_{1}^{\prime}(s) V_{2}(s)+k_{1}(s) V_{2}^{\prime}(s) \\
& =k_{1}^{\prime}(s) V_{2}(s)+k_{1}(s)\left(D_{V_{1}} V_{2}(s)-\frac{1}{2}\left[V_{1}(s), V_{2}(s)\right]\right) \\
& =k_{1}^{\prime}(s) V_{2}(s)+k_{1}(s)\left(-k_{1}(s) V_{1}(s)+k_{3}(s)-\bar{k}_{2}(s) V_{3}(s)\right) \\
& =-k_{1}^{2}(s) V_{1}(s)+k_{1}^{\prime}(s) V_{2}(s)+k_{1}(s)\left(k_{2}(s)-\bar{k}_{2}(s)\right) V_{3}(s)
\end{aligned}
$$

Also, we have the following:

$$
\begin{aligned}
\alpha^{\prime \prime \prime \prime}(s)= & -2 k_{1}(s) k_{1}^{\prime}(s) V_{1}(s)+k_{1}^{\prime \prime}(s) V_{2}(s)+\left(k_{1}(s) k_{2}(s)-k_{1}(s) \bar{k}_{2}(s)\right)^{\prime} V_{3}(s) \\
& -k_{1}^{2}(s) V_{1}^{\prime}(s)+k_{1}^{\prime}(s) V_{2}^{\prime}(s)+k_{1}(s)\left(k_{2}(s)-\bar{k}_{2}(s)\right) V_{3}^{\prime}(s) \\
= & -2 k_{1}(s) k_{1}^{\prime}(s) V_{1}(s)+k_{1}^{\prime \prime}(s) V_{2}(s)+\left(k_{1}(s) k_{2}(s)-k_{1}(s) \bar{k}_{2}(s)\right)^{\prime} V_{3}(s) \\
& -k_{1}^{2}(s)\left(D_{V_{1}} V_{1}(s)-\frac{1}{2}\left[V_{1}(s), V_{1}(s)\right]\right)+k_{1}^{\prime}(s)\left(D_{V_{1}} V_{2}(s)-\frac{1}{2}\left[V_{1}(s), V_{2}(s)\right]\right) \\
& +k_{1}(s)\left(k_{2}(s)-\bar{k}_{2}(s)\right)\left(D_{V_{1}} V_{3}(s)-\frac{1}{2}\left[V_{1}(s), V_{3}(s)\right]\right)
\end{aligned}
$$


Journal of Applied Mathematics

$$
\begin{aligned}
=-3 k_{1}(s) k_{1}^{\prime}(s) V_{1}(s)+[ & k_{1}^{\prime \prime}(s)-k_{1}^{3}(s)-k_{1}(s) k_{2}^{2}(s)+2 k_{1}(s) k_{2}(s) \bar{k}_{2}(s) \\
& \left.-k_{1}(s) \bar{k}_{2}^{2}(s)\right] V_{2}(s)+\left(2 k_{1}^{\prime}(s) \tau_{1}(s)+k_{1}(s) \tau_{1}^{\prime}(s)\right) V_{3}(s) .
\end{aligned}
$$

Notation. Let we put

$$
\begin{aligned}
N_{1}(s)= & k(s) V_{2}(s) \\
N_{2}(s)= & k_{1}^{\prime}(s) V_{2}(s)+k_{1}(s) \tau_{1}(s) V_{3}(s) \\
N_{3}(s)= & {\left[k_{1}^{\prime \prime}(s)-k_{1}^{3}-k_{1}(s) k_{2}^{2}(s)+2 k_{1}(s) k_{2}(s) \bar{k}_{2}(s)-k_{1}(s) \bar{k}_{2}^{2}(s)\right] V_{2}(s) } \\
& +\left(2 k_{1}^{\prime}(s) \tau_{1}(s)+k_{1}(s) \tau_{1}^{\prime}(s)\right) V_{3}(s) .
\end{aligned}
$$

\section{Curves of $\mathrm{AW}(\mathbf{k})$-Type}

In this section, we consider the properties of curves of AW(k)-type in the Lie group G.

Definition 3.1 (see, cf. [13]). The Frenet curves of osculating order 3 are

(i) of type weak $\mathrm{AW}(2)$ if they satisfy

$$
N_{3}(s)=\left\langle N_{3}(s), N_{2}^{*}(s)\right\rangle N_{2}^{*}(s),
$$

(ii) of type weak AW(3) if they satisfy

$$
N_{3}(s)=\left\langle N_{3}(s), N_{1}^{*}(s)\right\rangle N_{1}^{*}(s),
$$

where

$$
\begin{aligned}
N_{1}^{*}(s) & =\frac{N_{1}(s)}{\left\|N_{1}(s)\right\|^{\prime}} \\
N_{2}^{*}(s) & =\frac{N_{2}(s)-\left\langle N_{2}(s), N_{1}^{*}(s)\right\rangle N_{1}^{*}(s)}{\left\|\left\langle N_{2}(s), N_{1}^{*}(s)\right\rangle N_{1}^{*}(s)\right\|} .
\end{aligned}
$$

Definition 3.2 (see [8]). The Frenet curves of osculating order 3 are

(i) of type AW(1) if they satisfy $N_{3}(s)=0$,

(ii) of type $\mathrm{AW}(2)$ if they satisfy

$$
\left\|N_{2}(s)\right\|^{2} N_{3}(s)=\left\langle N_{3}(s), N_{2}(s)\right\rangle N_{2}(s),
$$


(iii) of type $\mathrm{AW}(3)$ if they satisfy

$$
\left\|N_{1}(s)\right\|^{2} N_{3}(s)=\left\langle N_{3}(s), N_{1}(s)\right\rangle N_{1}(s)
$$

From the definitions of type $\mathrm{AW}(\mathrm{k})$, we can obtain the following propositions.

Proposition 3.3. Let $\alpha$ be a Frenet curve of osculating order 3. Then $\alpha$ is of weak AW(2)-type if and only if

$$
k_{1}^{\prime \prime}(s)-k_{1}^{3}(s)-k_{1}(s) k_{2}^{2}(s)+2 k_{1}(s) k_{2}(s) \bar{k}_{2}(s)-k_{1}(s) \bar{k}_{2}^{2}(s)=0
$$

Proposition 3.4. Let $\alpha$ be a Frenet curve of osculating order 3. Then $\alpha$ is of weak AW(3)-type if and only if

$$
2 k_{1}^{\prime}(s) \tau_{1}(s)+k_{1}(s) \tau_{1}^{\prime}(s)=0
$$

Proposition 3.5. Let $\alpha$ be a Frenet curve of osculating order 3. Then $\alpha$ is of AW(1)-type if and only if

$$
\begin{gathered}
k_{1}^{\prime \prime}(s)-k_{1}^{3}(s)-k_{1}(s) k_{2}^{2}(s)+2 k_{1}(s) k_{2}(s) \bar{k}_{2}(s)-k_{1}(s) \bar{k}_{2}^{2}(s)=0, \\
k_{1}^{2}(s) \tau_{1}(s)=c
\end{gathered}
$$

where $c$ is a constant.

Proposition 3.6. Let $\alpha$ be a Frenet curve of osculating order 3. Then $\alpha$ is of type AW(2) if and only if

$$
\begin{aligned}
& k_{1}^{\prime}(s)\left(2 k_{1}^{\prime}(s) \tau_{1}(s)+k_{1}(s) \tau_{1}^{\prime}(s)\right) \\
& \quad=k_{1}(s) \tau_{1}(s)\left(k_{1}^{\prime \prime}(s)-k_{1}^{3}(s)-k_{1}(s) k_{2}^{2}(s)+2 k_{1}(s) k_{2}(s) \bar{k}_{2}(s)-k_{1}(s) \bar{k}_{2}^{2}(s)\right)=0 .
\end{aligned}
$$

Proposition 3.7. Let $\alpha$ be a Frenet curve of osculating order 3. Then $\alpha$ is of type AW(3) if and only if

$$
k_{1}^{2}(s) \tau_{1}(s)=c,
$$

where $c$ is a constant.

\section{General Helices of AW(k)-Type}

In this section, we study general helices of AW(k)-type in the Lie group $G$ with a bi-invariant metric and characterize these curves.

Definition 4.1 (see [6]). Let $\alpha: I \rightarrow G$ be a parameterized curve. Then $\alpha$ is called a general helix if it makes a constant angle with a left-invariant vector field. 
Note that in the definition the left-invariant vector field may be assumed to be with unit length, and if the curve $\alpha$ is parametrized by arc-length $s$, then we have

$$
\left\langle\alpha^{\prime}(s), X\right\rangle=\cos \theta
$$

for $X \in \mathfrak{g}$, where $\theta$ is a constant.

If $G$ is a commutative group $\mathbb{R}^{3}$, then Definition 4.1 reduces to the classical definition (see [14]). Since a left-invariant vector field in $G$ is a Killing vector field, Definition 4.1 is similar to the definition given in [1].

Theorem 4.2 (see [6]). A curve of osculating order 3 in G is a general helix if and only if

$$
\tau_{1}=c k_{1}
$$

where $c$ is a constant.

From (4.2), a curve with $k_{1} \neq 0$ is a general helix if and only if $\left(\tau_{1} / k_{1}\right)(s)=$ constant. As a Euclidean sense, if both $k_{1}(s) \neq 0$ and $\tau_{1}(s)$ are constants, it is a cylindrical helix. We call such a curve a circular helix.

Theorem 4.3. Let $\alpha$ be a Frenet curve of osculating order 3. Then $\alpha^{\prime \prime}(s), \alpha^{\prime \prime \prime}(s)$, and $\alpha^{\prime \prime \prime \prime}(s)$ are linearly dependent if and only if $\alpha(s)$ is general helix.

Proof. If $\alpha^{\prime \prime}(s), \alpha^{\prime \prime \prime}(s)$, and $\alpha^{\prime \prime \prime \prime}(s)$ are linearly dependent, then the following equation holds:

$$
\left|\begin{array}{ccc}
0 & k_{1} & 0 \\
-k_{1}^{2} & k_{1}^{\prime} & k_{1} \tau_{1} \\
-3 k_{1} k_{1}^{\prime} & k_{1}^{\prime \prime}-k_{1}^{3}-k_{1} k_{2}^{2}+2 k_{1} k_{2} \bar{k}_{2}-k_{1} \bar{k}_{2}^{2} & 2 k_{1}^{\prime} \tau_{1}+k_{1} \tau_{1}^{\prime}
\end{array}\right|=0
$$

By a direct computation, we have

$$
k_{1} \tau_{1}^{\prime}-k_{1}^{\prime} \tau_{1}=0
$$

it follows that

$$
\frac{d}{d s}\left(\frac{\tau_{1}}{k_{1}}\right)=0
$$

Thus, $\tau_{1} / k_{1}=$ constant; that is, $\alpha$ is general helix. The converse statement is trivial.

Theorem 4.4. Let $\alpha$ be a general helix of osculating order 3. Then $\alpha$ is of weak AW(3)-type if and only if $\alpha$ is a circular helix.

Proof. From (3.7) and (4.2), we can obtain that $k_{1}=$ constant; it follows that $\tau_{1}=$ constant. Thus, $\alpha$ is a circular helix. The converse statement is trivial. 
Theorem 4.5. A general helix of type AW(2) has Frenet curvatures

$$
k_{1}(s)=\frac{1}{\sqrt{-\left(1+c^{2}\right) s^{2}+d_{1} s+d_{2}}}, \quad \tau_{1}(s)=c k_{1}(s),
$$

where $c, d_{1}$, and $d_{2}$ are constants.

Proof. If $\alpha$ is a general helix of type AW(2), then from (3.9) and (4.2) we have

$$
\begin{gathered}
k_{1}^{\prime}(s)\left(2 k_{1}^{\prime}(s) \tau_{1}(s)+k_{1}(s) \tau_{1}^{\prime}(s)\right) \\
=k_{1}(s) \tau_{1}(s)\left(k_{1}^{\prime \prime}(s)-k_{1}^{3}(s)-k_{1}(s) k_{2}^{2}(s)+2 k_{1}(s) k_{2}(s) \bar{k}_{2}(s)-k_{1}(s) \bar{k}_{2}^{2}(s)\right)=0 \\
\frac{\tau_{1}(s)}{k_{1}(s)}=c
\end{gathered}
$$

where $c$ is a constant.

Combining (4.7) and (4.8), we have

$$
k_{1}(s) k_{1}^{\prime \prime}(s)-3\left(k_{1}^{\prime}(s)\right)^{2}-\left(1+c^{2}\right) k_{1}^{4}(s)=0
$$

To solve this differential equation, we take

$$
k_{1}(s)=x .
$$

Then, (4.9) can be rewritten as the form

$$
x \frac{d^{2} x}{d s^{2}}-3\left(\frac{d x}{d s}\right)^{2}=\left(1+c^{2}\right) x^{4}
$$

Let us put

$$
x=y^{p} .
$$

Then (4.11) becomes

$$
p y^{2 p-1} \frac{d^{2} y}{d s^{2}}-p(2 p+1) y^{2 p-2}\left(\frac{d y}{d s}\right)^{2}=\left(1+c^{2}\right) y^{4 p}
$$

If we choose $p=-1 / 2$, then the above equation is

$$
\frac{d^{2} y}{d s^{2}}=-2\left(1+c^{2}\right)
$$


Journal of Applied Mathematics

its general solution is given by

$$
y=-\left(1+c^{2}\right) s^{2}+d_{1} s+d_{2}
$$

where $d_{1}$ and $d_{2}$ are constants.

Thus, we have

$$
k_{1}(s)=\frac{1}{\sqrt{-\left(1+c^{2}\right) s^{2}+d_{1} s+d_{2}}}
$$

so, the theorem is proved.

Corollary 4.6. There exists no a circular helix of osculating order 3 of type AW(2) in G.

Theorem 4.7. Let $\alpha$ be a general helix of osculating order 3. Then $\alpha$ is of type AW(3) if and only if $\alpha$ is a circular helix.

Proof. Suppose that $\alpha$ is a general helix of type AW(3). Combining (3.10) and (4.2) we find $k_{1}^{3}(s)=1$, that is, $k_{1}(s)=1$. From this $\tau_{1}(s)=c$. Thus, $\alpha$ is a circular helix.

Theorem 4.8. Let $\alpha$ be a curve of osculating order 3. There exists no a general helix of type AW(1).

Proof. We assume that $\alpha$ is a general helix of type AW(1). Then from (3.8) and (4.2) we have

$$
\begin{gathered}
k_{1}^{\prime \prime}(s)-k_{1}^{3}(s)-k_{1}(s) k_{2}^{2}(s)+2 k_{1}(s) k_{2}(s) \bar{k}_{2}(s)-k_{1}(s) \bar{k}_{2}^{2}(s)=0, \\
k_{1}^{2}(s) \tau_{1}(s)=c, \\
\tau_{1}(s)=c k_{1}(s) .
\end{gathered}
$$

From (4.18) and (4.19), we have

$$
k_{1}(s)=1
$$

Thus, (4.17) becomes

$$
k_{2}^{2}(s)-2 k_{2}(s) \bar{k}_{2}(s)+\bar{k}_{2}^{2}(s)=-1,
$$

equivalently to

$$
\left(k_{2}(s)-\bar{k}_{2}(s)\right)^{2}=-1 .
$$

It is impossible, so the theorem is proved. 


\section{Acknowledgments}

This paper was supported by the Basic Science Research Program through the National

Research Foundation of Korea (NRF) funded by the Ministry of Education, Science and Technology (2012R1A1A2003994).

\section{References}

[1] M. Barros, "General helices and a theorem of Lancret," Proceedings of the American Mathematical Society, vol. 125, no. 5, pp. 1503-1509, 1997.

[2] A. Şenol and Y. Yayli, "LC helices in space forms," Chaos, Solitons and Fractals, vol. 42, no. 4, pp. 21152119, 2009.

[3] J. Arroyo, M. Barros, and O. J. Garay, "Models of relativistic particle with curvature and torsion revisited," General Relativity and Gravitation, vol. 36, no. 6, pp. 1441-1451, 2004.

[4] J. V. Beltran and J. Monterde, "A characterization of quintic helices," Journal of Computational and Applied Mathematics, vol. 206, no. 1, pp. 116-121, 2007.

[5] C. Camcı, K. İlarslan, L. Kula, and H. H. Hacısalihoğlu, "Harmonic curvatures and generalized helices in $E^{n}, "$ Chaos, Solitons E Fractals, vol. 40, no. 5, pp. 2590-2596, 2009.

[6] Ü. Çiftçi, "A generalization of Lancret's theorem," Journal of Geometry and Physics, vol. 59, no. 12, pp. 1597-1603, 2009.

[7] R. T. Farouki, C. Y. Han, C. Manni, and A. Sestini, "Characterization and construction of helical polynomial space curves," Journal of Computational and Applied Mathematics, vol. 162, no. 2, pp. 365-392, 2004.

[8] K. Arslan and A. West, "Product submanifolds with pointwise 3-planar normal sections," Glasgow Mathematical Journal, vol. 37, no. 1, pp. 73-81, 1995

[9] M. Külahc1, M. Bektaş, and M. Ergüt, "On harmonic curvatures of a Frenet curve in Lorentzian space," Chaos, Solitons and Fractals, vol. 41, no. 4, pp. 1668-1675, 2009.

[10] C. Özgür and F. Gezgin, “On some curves of $A W(k)$-type," Differential Geometry—Dynamical Systems, vol. 7, pp. 74-80, 2005.

[11] M. Külahci, M. Bektaş, and M. Ergüt, "Curves of $A W(k)$-type in 3-dimensional null cone," Physics Letters A, vol. 371, no. 4, pp. 275-277, 2007.

[12] N. do Espírito-Santo, S. Fornari, K. Frensel, and J. Ripoll, "Constant mean curvature hypersurfaces in a Lie group with a bi-invariant metric," Manuscripta Mathematica, vol. 111, no. 4, pp. 459-470, 2003.

[13] K. Arslan and C. Özgür, "Curves and surfaces of $A W(k)$ type," in Geometry and Topology of Submanifolds, IX (Valenciennes/Lyon/Leuven, 1997), pp. 21-26, World Scientific, River Edge, NJ, USA, 1999.

[14] D. J. Struik, Lectures on Classical Differential Geometry, Dover, New York, NY, USA, 1988. 


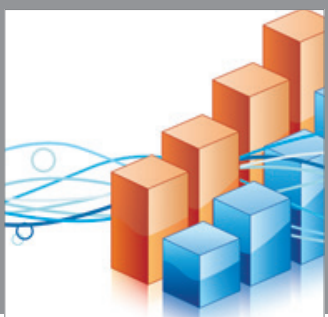

Advances in

Operations Research

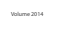

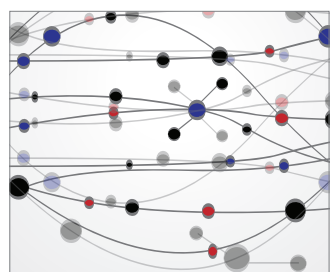

\section{The Scientific} World Journal
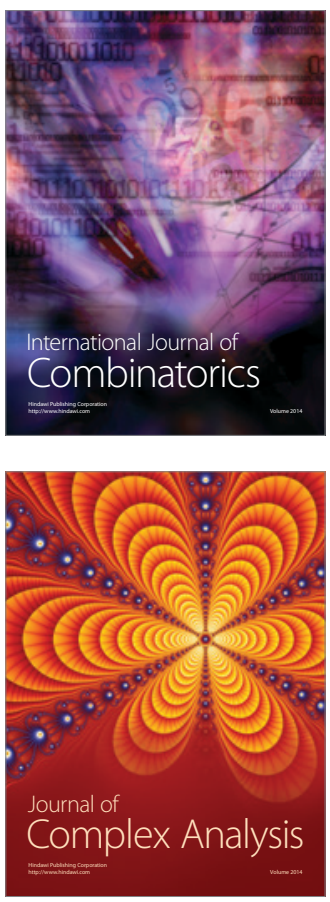

International Journal of

Mathematics and

Mathematical

Sciences
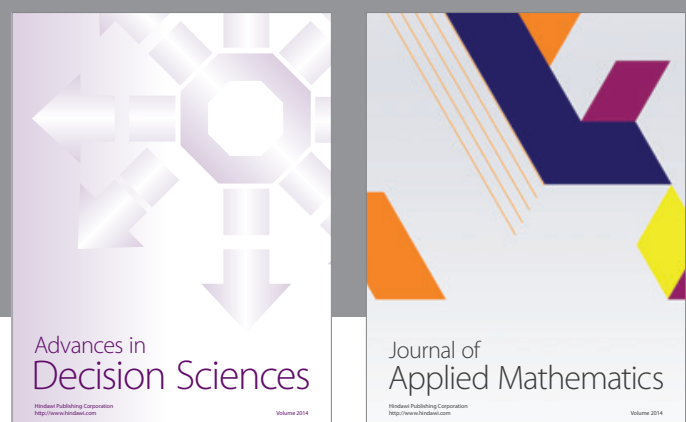

Journal of

Applied Mathematics
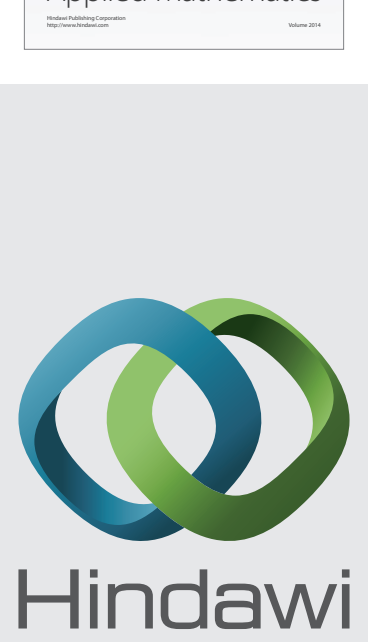

Submit your manuscripts at http://www.hindawi.com
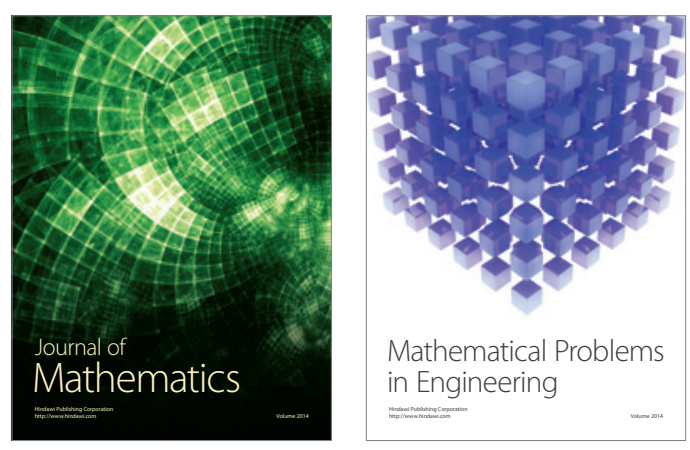

Mathematical Problems in Engineering
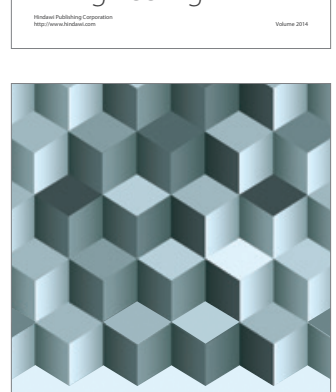

Journal of

Function Spaces
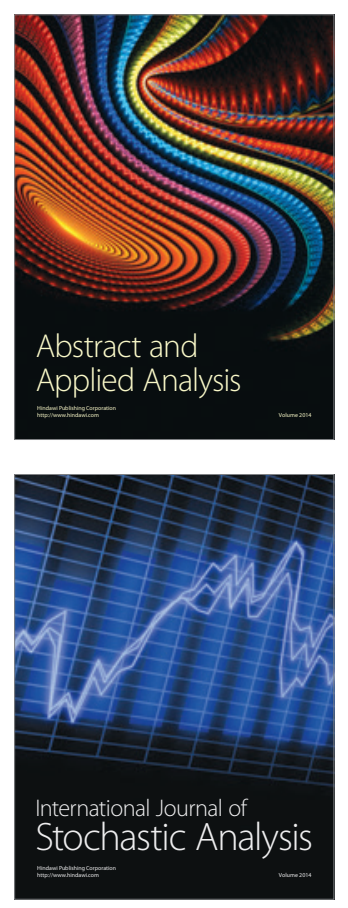

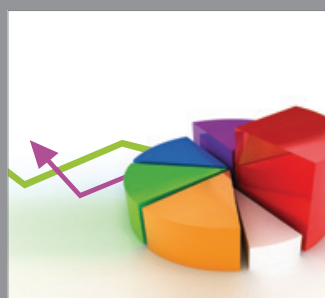

ournal of

Probability and Statistics

Promensencen
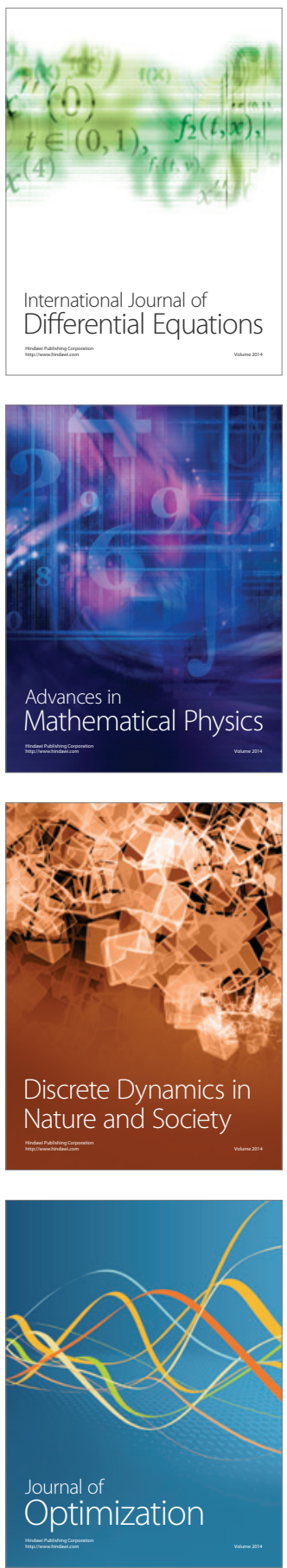